\title{
SPARC Expression as a Prognostic Marker in Pancreatic Ductal Adenocarcinoma
}

\author{
Jianxing Zhang ${ }^{1 *}$, Peiyu $\mathrm{Ji}^{2}$, Xin Fan ${ }^{1}$, Xiaoyan $\mathrm{Ma}^{3}$, Xuqing Wang1, Zhengfa Mao ${ }^{{ }^{*}}$ \\ ${ }^{1}$ Department of General Surgery, Affiliated Hospital of Jiangsu University, Zhenjiang, China \\ ${ }^{2}$ College of Medicine, Jiangsu University, Zhenjiang, China \\ ${ }^{3}$ Department of Gynecology and Obstetrics, Affiliated Hospital of Jiangsu University, Zhenjiang, China \\ Email: *89092246@qq.com, *jdh127880@sina.com
}

How to cite this paper: Zhang, J.X., Ji, P.Y., Fan, X., Ma, X.Y., Wang, X.Q. and Mao, Z.F. (2018) SPARC Expression as a Prognostic Marker in Pancreatic Ductal Adenocarcinoma. Open Journal of Gastroenterology, 8, 119-129.

https://doi.org/10.4236/ojgas.2018.84013

Received: March 6, 2018

Accepted: April 8, 2018

Published: April 11, 2018

Copyright (c) 2018 by authors and Scientific Research Publishing Inc. This work is licensed under the Creative Commons Attribution International License (CC BY 4.0).

http://creativecommons.org/licenses/by/4.0/

\begin{abstract}
Background: The aim is to investigate whether secreted protein acidic and rich in cysteine (SPARC) expression could be considered as an independent prognostic factor in pancreatic ductal adenocarcinoma (PDAC). Methods: The expression of SPARC in the tumor species was detected by immunochemistry and the effect of SPARC on the prognosis was analyzed by Kaplan-Meier and Cox proportional hazard models. Results: Positive SPARC expressions recognized in tumor and stroma were $31.33 \%$ and $64.66 \%$ respectively. The expression of SPARC in stroma was closely correlated with differentiation, vascular invasion and was an indicator for predicting poor outcome after surgery in Kaplan-Meier analysis. Univariate analysis and multivariate analysis showed the following factors to be significant: perineural invasion (HR $=2.098,95 \% \mathrm{CI}: 1.144-3.847)$ and stroma positive expression of SPARC (HR $=2.120,95 \%$ CI: 1.307 - 3.440). Conclusions: High expression of SPARC in stroma was a promising pathological marker to predict the poor outcome in patients with pancreatic ductal adenocarcinoma.
\end{abstract}

\section{Keywords}

Pancreatic Adenocarcinoma, SPARC, Prognosis, Surgery

\section{Introduction}

Pancreatic ductal adenocarcinoma (PDAC) is one of the leader causes of cancer-related mortality in the world [1]. At the moment of diagnosis, most patients presented with locally advanced or metastatic status and were not considered as surgical candidate. Even for patients who were candidates for potentially curative resection, 5 -year survival is poor, reported to be of $15 \%$ to $25 \%$ [2]. Al- 
though the advancement in radiologic imaging, surgical treatment and novel drugs for adjuvant therapies have contributed to the outcomes improvement for those patients, significant progress hasn't been demonstrated.

It is crucial to detect the accurate staging and find the novel clinically useful prognostic factors for improving the prognosis. Some clinic parameters have been clearly considered as prognostic factors such as tumor size, degree of histological differentiation and resection margin status [3]. But all of the above detections were post-surgery and could not be used as predictive factor preoperation.

Genomic alteration and the epigenetic changes have been validated involved in the development of tumor. It is well known that these changes are considered as an early event during the carcinogenesis and can predict the occurrence of tumor. The secreted protein acidic and rich in cysteine (SPARC), is a calcium binding glycoprotein that shows a high degree of interspecies sequence conservation and plays an important role in various cellular progression including tissue remodeling, wound repair, morphogenesis, cellular differentiation, cell proliferation, cell migration, and angiogenesis [4] [5] [6]. The expression pattern of SPARC is various in different types of tumors. It highly expresses in some human carcinomas and promotes the malignant biologic character; while it is down regulated in other types and the low expression is associated with poor prognosis [7] [8] [9] [10] [11]. Notable, in some types of tumor characterize with strong desmoplastic reaction, SPARC is predominantly located in the interface of between the stroma and the tumor cells, which indicates that SPARC may take part in the carcinogenesis [8] [12] [13]. A study has been revealed that treating human ovarian cancer cells with exogenous SPARC could inhibit cell proliferation and induce apoptosis [14]. In addition, in xenograft tumor model in nude mice, forced expression of SPARC in ovarian cancer cells could reduce tumor cells tumorigenicity, implying that SPARC has a tumor-suppressor function. Furthermore, SPARC could improve the potential of invasive in prostate and breast cancer cells in vitro [15] and promote invasion of glioma in vivo [16]. Several studies have found that it could be considered as an independent prognostic factor in pancreatic carcinoma [17] [18].

The aim of this retrospective study was to delineate the clinicopathologic parameter and expression pattern of SPARC and the association between them in the patients diagnosed with pancreatic ductal adenocarcinoma.

\section{Materials and Methods}

\section{Demographic features of 150 patients with PDAC}

From the PDAC registration of the Department of General Surgery, Affiliated Hospital of Jiangsu University, PDAC patients recruited for the current study undergoing radical surgery between 2005 and 2015 were based on the availability of pathological samples for slides. Negative margin of the resection detected by microscopy was defined as curative resection. Surgical mortality defined as death of 
patients within the first month was excluded the survival analysis. The tumors were staged according to the International Union Against Cancer tumor-node-metastasis (TNM) classification, 7th edition. The protocol of this study was reviewed and approved by the institutional ethics committee. Informed consent was obtained for every specimen examined.

\section{Immunohistochemical study of SPARC}

Slides stained with hematoxylin/eosin from each patient were reviewed. Specimens from all PDAC patients had been fixed in formalin and embedded in paraffin. Formalin-fixed paraffin-embedded tissue sections (5 $\mu \mathrm{m}$ thick) were stained using the immunoperoxidase method with avidin-biotin complex as described previously [19]. The primary antibody against SPARC (life science, $\mathrm{H} 95031 \mathrm{M})$ at 1:500 dilutions incubated with slides overnight at $4^{\circ} \mathrm{C}$. The slides were washed thrice in Tris-buffered saline supplement with Tween-20 for 5 min each before visualization with the Envision Plus Detection Kit (DAKO, Carpinteria, CA) followed the manufacturer's protocol. The absence of nonspecific staining was confirmed by the control staining omitting the primary antibody. After washing thrice in Tris-buffered saline supplement with Tween-20 for 5 min each, the slides were mounted. Nuclei were counterstained with hematoxylin. Immunohistochemical results were judged by three pathologists who were unaware of the clinical data.

SPARC immunostaining was stratified as previous described [20]: tumor negative/stroma negative, tumor positive/stroma negative, tumor negative/stroma positive, and tumor positive/stroma positive. The staining results were judged by German semiquantitatives scoring system which considers both the stain intensity and the area extent and the definition of low and high expression as previous described [21].

\section{Statistical analysis}

Statistical analysis of correlation between the SPARC expression and the clinic parameter was performed using $\chi^{2}$ test. The overall survival (OS) was estimated using Kaplan-Meier method. A univariable analysis of the clinicopathological variables was performed using the log-rank test. A multivariate regression was performed using the Cox proportional hazards model. All analyses were performed using the statistical software SPSS (version 16.0 for windows; SPSS Inc., Chicago, IL, USA). All $p$ value derived from two-tailed test and $p<0.05$ was considered statistically significant.

\section{Results}

\section{SPARC staining of human PDAC specimens in relation to clinic parame- ter features}

In the IHC analysis, we can see the main staining of SPARC was located in the stroma and weak in tumor cells as previous results [20]. In stroma, SPARC expression was mostly obviously seen in the cytoplasm of peritumoral fibroblasts while the staining was weak or even absent in the stroma distant from the cancer 
cells. The extracellular matrix and other inflammatory cells did not show the expression of SPARC. Within total 150 subjects, 30 (20\%) cases were classified as tumor negative/stroma negative, $23(15.3 \%)$ cases as tumor positive/stroma negative, $73(48.7 \%)$ cases as tumor negative/stroma positive and $24(16 \%)$ cases as tumor positive/stroma positive (Figure 1). Parameters association was compared for all the 150 patients according to their stromal SPARC status (Table 1). SPARC positive expression was significantly related with vascular invasion, differentiated status $(p<0.05)$ while irrespective of sex, age, perineural invasion, lymph nodal invasion $(p>0.05)$.

Table 1. Association between SPARC expression and clinicopathological characteristics of patients with PDAC $(n=150)$.

\begin{tabular}{|c|c|c|c|c|}
\hline \multirow{2}{*}{ Characteristic } & \multirow{2}{*}{$\begin{array}{c}\text { No. of } \\
\text { Patients }\end{array}$} & \multicolumn{2}{|c|}{ SPARC expression (stroma) } & \multirow{2}{*}{$\chi^{2}$ test } \\
\hline & & negative & positive & \\
\hline \multicolumn{5}{|c|}{ Sex } \\
\hline Male & 86 & 31 & 55 & \multirow{2}{*}{$P=0.832$} \\
\hline Female & 64 & 22 & 42 & \\
\hline \multicolumn{5}{|c|}{ Age (years) } \\
\hline$<60$ & 72 & 28 & 44 & \multirow{2}{*}{$P=0.381$} \\
\hline$\geq 60$ & 78 & 25 & 53 & \\
\hline \multicolumn{5}{|c|}{ Tumor position } \\
\hline head and neck & 111 & 40 & 71 & \multirow{2}{*}{$P=0.761$} \\
\hline body and tail & 39 & 13 & 26 & \\
\hline \multicolumn{5}{|c|}{ Differentiation } \\
\hline Well & 5 & 5 & 0 & \multirow{3}{*}{$P=0.008^{*}$} \\
\hline Moderately & 96 & 33 & 63 & \\
\hline Poorly & 49 & 15 & 34 & \\
\hline \multicolumn{5}{|c|}{ TNM stage } \\
\hline $\mathrm{I}+\mathrm{II}$ & 89 & 32 & 57 & \multirow{2}{*}{$P=0.847$} \\
\hline $\mathrm{III}+\mathrm{IV}$ & 61 & 21 & 40 & \\
\hline \multicolumn{5}{|c|}{ perineural invasion } \\
\hline Absent & 38 & 15 & 23 & \multirow{2}{*}{$P=0.537$} \\
\hline Present & 112 & 38 & 74 & \\
\hline \multicolumn{5}{|c|}{ Vascular invasion } \\
\hline Absent & 133 & 52 & 81 & \multirow{2}{*}{$P=0.006^{\star}$} \\
\hline Present & 17 & 1 & 16 & \\
\hline \multicolumn{5}{|c|}{ LN metastasis } \\
\hline Absent & 90 & 32 & 58 & \multirow{2}{*}{$P=0.994$} \\
\hline Present & 60 & 21 & 39 & \\
\hline
\end{tabular}

TNM, tumor-node-metastasis. LN: lymph node ${ }^{*} P<0.05$. 


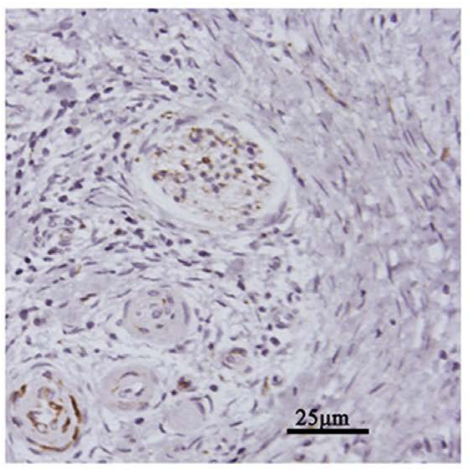

(a)

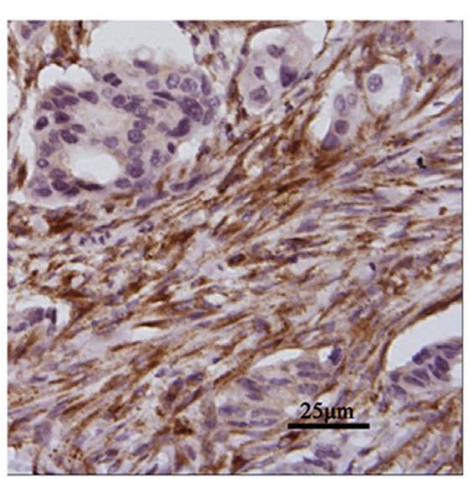

(c)

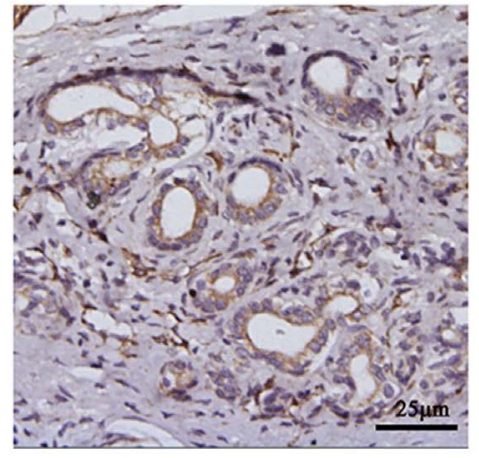

(b)

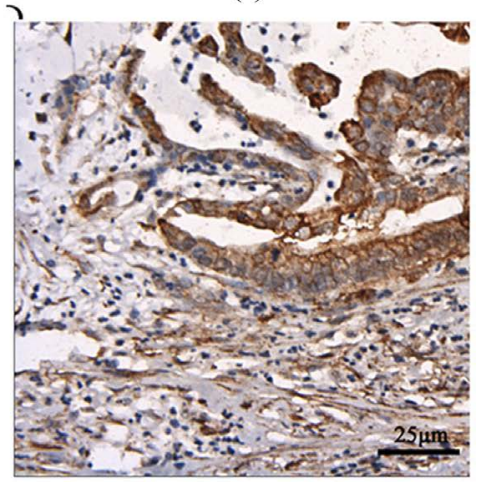

(d)

Figure 1. The expression of SPARC detected by immunohistochemistry in pancreatic ductal adenocarcinomas samples. (a) SPARC expression: cancer negative, stromal expression negative; (b) SPARC expression: cancer positive, stromal expression negative; (c) SPARC expression: cancer negative, stromal expression positive; and (d) SPARC expression: cancer positive, stromal expression positive.

\section{Analyses for survival and prognosis for PDAC patients with SPARC}

A total of 111 PDAC patients who had undergone radical surgery were enrolled in the survival analysis. The patients (62 men and 49 women) with a median age of 60.99 years were followed-up until death after surgery. The OS rates at 3 years were $24.3 \%(27 / 111)$.

Kaplan-Meier test identified the following factors as adverse influences on the OS rate of 111 PDAC patients who had undergone radical surgery: perineural invasion $(p=0.009)$, TNM stage $(p=0.04)$, lymph node metastasis $(p=0.001)$ and SPARC expression stromal positive $(p=0.001)$. In contrast, SPARC expression within the malignant epithelial cells was not significantly associated with prognosis (log-rank $p=0.224$, Figure 2 ).

The univariate Cox proportional hazards regression shown that differentiation, TNM stage, perineural invasion, LN metastasis and positive stromal SPARC expression were considered as independent of tumor SPARC staining (Table 2). Meanwhile, multivariate Cox proportional hazard analysis demonstrated that positive stromal SPARC expression (HR $=2.120,95 \%$ CI: 1.307 $3.440, p=0.002)$ and perineural invasion $(\mathrm{HR}=2.098,95 \% \mathrm{CI}: 1.144-3.847, p=$ 
$0.017)$ independently predicted an adverse OS rate for PDAC patients after radical surgery (Table 3, Figure 3(a) and Figure 3(b)).

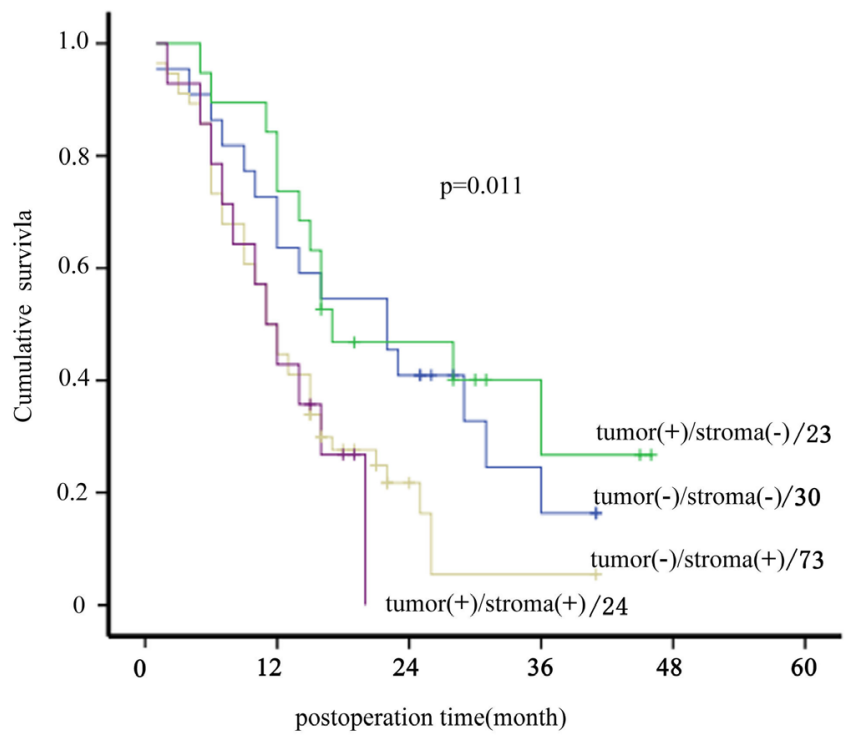

Figure 2. Kaplan-Meier survival curves stratified by tumor and stromal SPARC status of patients with pancreatic ductal adenocarcinoma after pancreaticoduodenectomy.

Table 2. Univariate analysis of prognostic factors for overall survival $(\mathrm{n}=111)$.

\begin{tabular}{ccccc}
\hline variable & SE & HR & $95.0 \%$ CI & $P$ \\
\hline Gender (male $v s$ female) & 0.220 & 1.075 & $0.699-1.653$ & 0.742 \\
Age $(\leq 60 v s>60)$ & 0.010 & 1.005 & $0.985-1.025$ & 0.639 \\
Tumor site (head vs body and tail) & 0.273 & 0.650 & $0.381-1.109$ & 0.114 \\
Differentiation (well, moderate and poor) & $\mathbf{0 . 1 9 9}$ & $\mathbf{1 . 4 8 2}$ & $\mathbf{1 . 0 0 2 - 2 . 1 9 1}$ & $\mathbf{0 . 0 4 9}$ \\
T stage (1, 2, 3) & 0.170 & 1.035 & $0.742-1.443$ & 0.838 \\
TNM stage (I, II, III) & $\mathbf{0 . 1 6 2}$ & $\mathbf{1 . 5 6 1}$ & $\mathbf{1 . 1 3 7 - 2 . 1 4 4}$ & $\mathbf{0 . 0 0 6}$ \\
Perinerual invasion (present $v \boldsymbol{s}$ absent) & $\mathbf{0 . 3 0 7}$ & $\mathbf{2 . 1 4}$ & $\mathbf{1 . 1 7 2 - 3 . 9 0 8}$ & $\mathbf{0 . 0 1 3}$ \\
Vascular invasion (present $v s$ absent) & 0.357 & 1.425 & $0.708-2.867$ & 0.321 \\
LN metastasis (present $v \boldsymbol{s}$ absent) & $\mathbf{0 . 2 2 1}$ & $\mathbf{2 . 1 0 6}$ & $\mathbf{1 . 3 6 5 - 3 . 2 5 1}$ & $\mathbf{0 . 0 0 1}$ \\
Tumor SPARC (positive $v s$ negative) & 0.246 & 0.758 & $0.469-1.227$ & 0.260 \\
Stroma SPARC (positive $v \boldsymbol{s}$ negative) & $\mathbf{0 . 2 4 5}$ & $\mathbf{2 . 1 5 1}$ & $\mathbf{1 . 3 3 0 - 3 . 4 7 7}$ & $\mathbf{0 . 0 0 2}$ \\
\hline
\end{tabular}

Table 3. Multivariate analysis of prognostic factors for overall survival $(\mathrm{n}=111)$.

\begin{tabular}{ccccc}
\hline Variable & SE & HR & $95.0 \%$ CI & $P$ \\
\hline Differentiation (well, moderate and poor) & 0.202 & 1.156 & $0.778-1.717$ & 0.473 \\
TNM stage (I, II, III) & 0.338 & 1.219 & $0.629-2.362$ & 0.558 \\
Perineural invasion (present $v \boldsymbol{s}$ absent) & $\mathbf{0 . 3 1 6}$ & $\mathbf{2 . 1 8 0}$ & $\mathbf{1 . 1 7 4 - 4 . 0 4 9}$ & $\mathbf{0 . 0 1 4}$ \\
LN metastasis (present $v s$ absent) & 0.5 & 1.605 & $0.603-4.275$ & 0.344 \\
Stroma SPARC (positive $v \boldsymbol{s}$ negative) & $\mathbf{0 . 2 5 8}$ & $\mathbf{2 . 1 1 9}$ & $\mathbf{1 . 2 7 9 - 3 . 5 1 1}$ & $\mathbf{0 . 0 0 4}$ \\
\hline
\end{tabular}




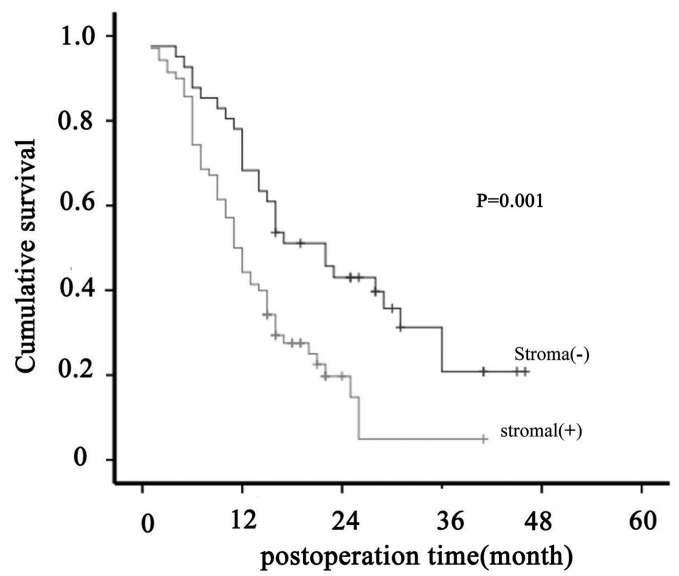

(a)

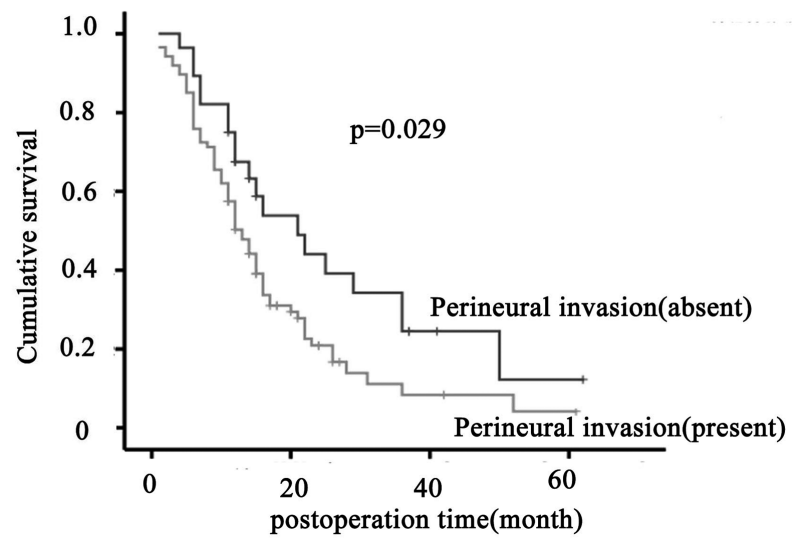

(b)

Figure 3. Comparison of Os rate of 111 PDAC patients who had undergone radical surgery in relation to (a) SPARC expression in stroma and (b) perineural invasion result. Abbreviations: OS, overall survival; PDAC,pancreatic ductla adenocarcinoma; SPARC, secreted protein acidic and rich in cysteine.

\section{Discussion}

In the present study, we demonstrated that the positive SPARC expression in pancreatic ductal adenocarcinoma mainly located in stroma while compared with the tumor cells, and were $64.66 \%$ (97/150) and 31.3\% (47/150), respectively. The high SPARC expression in stroma was associated with vascular invasion and differentiation in PDAC. Infante et al. reported this association in PDAC in the head of pancreases under Whipple procedure [20]. Previous research approves that through mediating MMPs activity, SPARC could enhance the invasive ability of prostate and breast cancer cells [22] [23]. Also in MCF7 breast cancer cells, increased motility and invasion induced by transfecting with c-Jun proto-oncogene could inhibit by the suppression of SPARC expression [24]. Shi et al. demonstrates that in glioma, SPARC delays tumor growth but promotes invasion in vivo [25]. It also finds that SPARC endows the ability of mediate growth factors involved in angiogenesis, such as platelet-derived growth factor 
(PDGF), fibroblast growth factor (FGF)-2, and vascular endothelial growth factor (VEGF) [26].

In the survival analysis, we demonstrated that high SPARC expression in the stroma is associated with poor prognosis in primary PDAC samples while the status in tumor cells was not. No matter the SPARC expression status in the tumor cells, the positive status in stroma was associated with the worst prognosis; notwithstanding median survival was both of 11 months. Meanwhile, the median survival time in tumor $(+) /$ stroma $(-)$ was slightly shorter than tumor $(-) /$ stroma $(-)$ although there was no statistic significance. The results consisted with previous research, but the underlying mechanism is unknown. Some studies show that transplanted tumors grow faster in xenograft tumor model in $\mathrm{SPARC}^{-/-}$mice [27] and the growth of pancreatic cancer cells is suppressed by exogenous SPARC protein in vitro [28]. These results suggest that a SPARC originated from peritumoral fibroblasts may play a role in inhibiting the growth of pancreatic cancer cells in vivo, in which SPARC expression losing frequently dues to the promoter methylation. In breast cancer, in cases with strong SPARC stromal staining, there is a trend towards increased disease-specific survival [29].

In the immunohistochemical analysis, high SPARC expression in stroma correlated with poor prognosis. Moreover, based on the multivariate analysis of OS, stroma high SPARC expression translated into an independently unfavorable prognostic factor. The involvement of SPARC in tumorigenesis of pancreatic cancer has been mentioned in several previous investigations. Early studies demonstrated that SPARC is a frequent target for aberrant methylation in pancreatic cancer [28] and exogenous SPARC protein has growth-suppressive activity on pancreatic cancer cells [30]. Pancreatic tumors grown orthotopically in Sparc-null $\left(\right.$ Sparc $\left.^{-1-}\right)$ mice are more metastatic than tumors grown inwild-type $\left(\right.$ Sparc $\left.^{+/+}\right)$littermates [31]. Some other studies found that the expression of SPARC by peritumoral fibroblasts portends a poorer prognosis for patients with pancreatic cancer [28]. Therefore, it suspects that SPARC may be a protective mechanism and acts as an indicator of invasive disease. Some reports have also revealed that high SPARC expression is related to poor prognosis in the following cancers: melanoma, gliomas [6] [16] [22]. The results of the present study were in agreement with these previous reports. Therefore, SPARC was expected to serve as a prognostic-predictive marker of PDAC.

We demonstrated that SPARC were over expressed in a large proportion of PADC tissues in stroma. In addition, high expressions of SPARC were correlated with the disease progression and poor clinical outcome of PDAC. Furthermore, SPARC was proved to be an independent prognostic factor for overall survival in patients with PDAC. Therefore, targeted inhibition of SPARC might be a new strategy for therapy of PDAC. Certainly, further strong supports from basic investigations are still needed.

\section{Conflict of Interest}

There is no conflict of interest. 


\section{Acknowledgements}

This work was sponsored by National Natural Science foundation of China, Grant numbers: 81502372; Natural Science Foundation of Jiangsu Province (Youth Fund), Grant numbers: BK20130475; Jiangsu Provincial Medical Youth Talent (QNRC2016838, QNRC2016839); Zhenjiang Science and Technology Pillar Program, Grant numbers: SH2014035; The Foundation for Young Scientists of affiliated Hospital of Jiangsu University (Grant numbers: JDFYRC2013009, JDFYRC2016002).

\section{References}

[1] Siegel, R.L., Miller, K.D. and Jemal, A. (2016) Cancer Statistics, 2016. CA: A Cancer Journal for Clinicians, 66, 7-30. https://doi.org/10.3322/caac.21332

[2] Wolfgang, C.L., Herman, J.M., Laheru, D.A., Klein, A.P., Erdek, M.A., Fishman, E.K. and Hruban, R.H. (2013) Recent Progress in Pancreatic Cancer. CA: A Cancer Journal for Clinicians, 63, 318-348. https://doi.org/10.3322/caac.21190

[3] Yan, T.T., Fu, X.L., Li, J., Bian, Y.N., Liu, D.J., Hua, R., Ren, L.L., Li, C.T., Sun, Y.W., Chen, H.Y., Fang, J.Y. and Hong, J. (2015) Downregulation of RPL15 May Predict Poor Survival and Associate with Tumor Progression in Pancreatic Ductal Adenocarcinoma. Oncotarget, 6, 37028-37042.

https://doi.org/10.18632/oncotarget.5939

[4] Reed, M.J. and Sage, E.H. (1996) SPARC and the Extracellular Matrix: Implications for Cancer and Wound Repair. Current Topics in Microbiology and Immunology, 213, 81-94. https://doi.org/10.1007/978-3-642-61107-0_6

[5] Hrabchak, C., Ringuette, M. and Woodhouse, K. (2008) Recombinant Mouse SPARC Promotes Parietal Endoderm Differentiation and Cardiomyogenesis in Embryoid Bodies. Biochemistry and Cell Biology, 86, 487-499.

https://doi.org/10.1139/O08-141

[6] Maloney, S.C., Marshall, J.C., Antecka, E., Orellana, M.E., Fernandes, B.F., Martins, C., Castiglione, E., DI. Cesare. S., Logan, P. and Burnier, Jr., M.N. (2009) SPARC Is Expressed in Human Uveal Melanoma and Its Abrogation Reduces Tumor Cell Proliferation. Anticancer Research, 29, 3059-3064.

[7] Shi, D., Jiang, K., Fu, Y., Fang, R., Liu, X.I. and Chen, J. (2016) Overexpression of SPARC Correlates with Poor Prognosis in Patients with Cervical Carcinoma and Regulates Cancer Cell Epithelial-Mesenchymal Transition, Oncology Letters, 11, 3251-3258. https://doi.org/10.3892/ol.2016.4399

[8] Kim, J.Y., Jeong, D., Ahn, T.S., Kim, H.J., Park, D.S., Park, S.Y., Bae, S.B., Lee, S., Lee, S.S., Lee, M.S., Cho, H.D. and Baek, M.J. (2013) Xpression of Secreted Protein Acidic and Rich in Cysteine in the Stroma of a Colorectal Carcinoma Is Associated with Patient Prognosis. Annals of Coloproctology, 29, 93-99.

https://doi.org/10.3393/ac.2013.29.3.93

[9] Wang, H.Y., Li, Y.Y., Shao, Q., Hou, J.H., Wang, F., Cai, M.B., Zeng, Y.X. and Shao, J.Y. (2012) Secreted Protein Acidic and Rich in Cysteine (SPARC) Is Associated with Nasopharyngeal Carcinoma Metastasis and Poor Prognosis. Journal of Translational Medicine, 10, 27. https://doi.org/10.1186/1479-5876-10-27

[10] Kato, Y., Nagashima, Y., Baba, Y., Kawano, T., Furukawa, M., Kubota, A., Yanoma, S., Imagawa-Ishiguro, Y., Satake, K., Taguchi, T., Hata, R., Mochimatsu, I., Aoki, I., Kameda, Y., Inayama, Y. and Tsukuda, M. (2005) Expression of SPARC in Tongue 
Carcinoma of Stage II Is Associated with Poor Prognosis: An Immunohistochemical Study of 86 Cases. International Journal of Molecular Medicine, 16, 263-268. https://doi.org/10.3892/ijmm.16.2.263

[11] Chin, D., Boyle, G.M., Williams, R.M., Ferguson, K., Pandeya, N., Pedley, J., Campbell, C.M., Theile, D.R., Parsons, P.G. and Coman, W.B. (2005) Novel Markers for Poor Prognosis in Head and Neck Cancer. International Journal of Cancer, 113, 789-797. https://doi.org/10.1002/ijc.20608

[12] Koukourakis, M.I., Giatromanolaki, A., Brekken, R.A., Sivridis, E., Gatter, K.C., Harris, A.L. and Sage, E.H. (2003) Enhanced Expression of SPARC/Osteonectin in the Tumor-Associated Stroma of Non-Small Cell Lung Cancer Is Correlated with Markers of Hypoxia/Acidity and with Poor Prognosis of Patients. Cancer Research, 63, 5376-5380.

[13] Podhajcer, O.L., Benedetti, L.G., Girotti, M.R., Prada, F., Salvatierra, E. and Llera, A.S. (2008) The Role of the Matricellular Protein SPARC in the Dynamic Interaction between the Tumor and the Host. Cancer and Metastasis Reviews, 27, 691-705. https://doi.org/10.1007/s10555-008-9146-7

[14] Yiu, G.K., Chan, W.Y., Ng, S.W., Chan, P.S., Cheung, K.K., Berkowitz, R.S. and Mok, S.C. (2001) SPARC (Secreted Protein Acidic and Rich in Cysteine) Induces Apoptosis in Ovarian Cancer Cells. The American Journal of Pathology, 159, 609-622. https://doi.org/10.1016/S0002-9440(10)61732-4

[15] Wong, S.Y., Crowley, D., Bronson, R.T. and Hynes, R.O. (2008) Analyses of the Role of Endogenous SPARC in Mouse Models of Prostate and Breast Cancer. Clinical \& Experimental Metastasis, 25, 109-118. https://doi.org/10.1007/s10585-007-9126-2

[16] Seno, T., Harada, H., Kohno, S., Teraoka, M., Inoue, A. and Ohnishi, T. (2009) Downregulation of SPARC Expression Inhibits Cell Migration and Invasion in $\mathrm{Ma}-$ lignant Gliomas. International Journal of Oncology, 34, 707-715. https://doi.org/10.3892/ijo_00000197

[17] Strimpakos, A.S., Syrigos, K.N. and Saif, M.W. (2013) Pharmacogenomics in Pancreatic Adenocarcinoma: New Data and Their Clinical Implications. JOP: Journal of the Pancreas, 14, 359-362.

[18] Mantoni, T.S., Schendel, R.R., Rodel, F., Niedobitek, G., Al-Assar, O., Masamune, A. and Brunner, T.B. (2008) Stromal SPARC Expression and Patient Survival after Chemoradiation for Non-Resectable Pancreatic Adenocarcinoma. Journal Cancer Biology \& Therapy, 7, 1806-1815. https://doi.org/10.4161/cbt.7.11.6846

[19] Sun, Z.J., Wang, Y., Cai, Z., Chen, P.P., Tong, X.J. and Xie, D. (2008) Involvement of Cyr61 in Growth, Migration, and Metastasis of Prostate Cancer Cells. British Journal of Cancer, 99, 1656-1667. https://doi.org/10.1038/sj.bjc.6604712

[20] Infante, J.R., Matsubayashi, H., Sato, N., Tonascia, J., Klein, A.P., Riall, T.A., Yeo, C., Iacobuzio-Donahue, C. and Goggins, M. (2007) Peritumoral Fibroblast SPARC Expression and Patient Outcome with Resectable Pancreatic Adenocarcinoma. Journal of Clinical Oncology, 25, 319-325. https://doi.org/10.1200/JCO.2006.07.8824

[21] Deng, Y.Z., Yao, F., Li, J.J., Mao, Z.F., Hu, P.T., Long, L.Y., Li, G., Ji, X.D., Shi, S., Guan, D.X., Feng, Y.Y., Cui, L., Li, D.S., Liu, Y., Du, X., Guo, M.Z., Xu, L.Y., Li, E.M., Wang, H.Y. and Xie, D. (2012) RACK1 Suppresses Gastric Tumorigenesis by Stabilizing the $\beta$-Catenin Destruction Complex. Gastroenterology, 142, 812-823. https://doi.org/10.1053/j.gastro.2011.12.046

[22] Kapinas, K., Lowther, K.M., Kessler, C.B., Tilbury, K., Lieberman, J.R., Tirnauer, 
J.S., Campagnola, P. and Delany, A.M. (2012) Bone Matrix Osteonectin Limits Prostate Cancer Cell Growth and Survival. Matrix Biology, 31, 299-307. https://doi.org/10.1016/j.matbio.2012.03.002

[23] Gilles, C., Bassuk, J.A., Pulyaeva, H., Sage, E.H., Foidart, J.M. and Thompson, E.W. (1998) SPARC/Osteonectin Induces Matrix Metalloproteinase 2 Activation in $\mathrm{Hu}$ man Breast Cancer Cell Lines. Cancer Research, 58, 5529-5536.

[24] Briggs, J., Chamboredon, S., Castellazzi, M., Kerry, J.A. and Bos, T.J. (2002) Transcriptional Upregulation of SPARC, in Response to c-Jun Overexpression, Contributes to Increased Motility and Invasion of MCF7 Breast Cancer Cells. Oncogene, 21, 7077-7091. https://doi.org/10.1038/sj.onc.1205857

[25] Shi, Q., Bao, S., Song, L., Wu, Q., Bigner, D.D., Hjelmeland, A.B. and Rich, J.N. (2007) Targeting SPARC Expression Decreases Glioma Cellular Survival and Invasion Associated with Reduced Activities of FAK and ILK Kinases. Oncogene, 26, 4084-4094. https://doi.org/10.1038/sj.onc.1210181

[26] Brekken, R.A. and Sage, E.H. (2001) SPARC, a Matricellular Protein: At the Crossroads of Cell-Matrix Communication. Matrix Biology, 19, 815-827. https://doi.org/10.1016/S0945-053X(00)00133-5

[27] Puolakkainen, P.A., Brekken, R.A., Muneer, S. and Sage, E.H. (2004) Enhanced Growth of Pancreatic Tumors in SPARC-Null Mice Is Associated with Decreased Deposition of Extracellular Matrix and Reduced Tumor Cell Apoptosis. Molecular Cancer Research, 2, 215-224.

[28] Sato, N., Fukushima, N., Maehara, N., Matsubayashi, H., Koopmann, J., Su, G.H., Hruban, R.H. and Goggins, M. (2003) SPARC/Osteonectin Is a Frequent Target for Aberrant Methylation in Pancreatic Adenocarcinoma and a Mediator of $\mathrm{Tu}$ mor-Stromal Interactions. Oncogene, 22, 5021-5030. https://doi.org/10.1038/sj.onc.1206807

[29] Nagai, M.A., Gerhard, R. Fregnani, J.H., Nonogaki, S., Rierger, R.B., Netto, M.M. and Soares, F.A. (2011) Prognostic Value of NDRG1 and SPARC Protein Expression in Breast Cancer Patients. Breast Cancer Research and Treatment, 126, 1-14. https://doi.org/10.1007/s10549-010-0867-2

[30] Guweidhi, A., Kleeff, J., Adwan, H., Giese, N.A., Wente, M.N., Giese, T., Buchler, M.W., Berger, M.R. and Friess, H. (2005) Osteonectin Influences Growth and Invasion of Pancreatic Cancer Cells. Annals of Surgery, 242, 224-234. https://doi.org/10.1097/01.sla.0000171866.45848.68

[31] Brekken, R.A., Puolakkainen, P., Graves, D.C., Workman, G., Lubkin, S.R. and Sage, E.H. (2003) Enhanced Growth of Tumors in SPARC Null Mice Is Associated with Changes in the ECM. The Journal of Clinical Investigation, 111, 487-495. https://doi.org/10.1172/JCI16804 